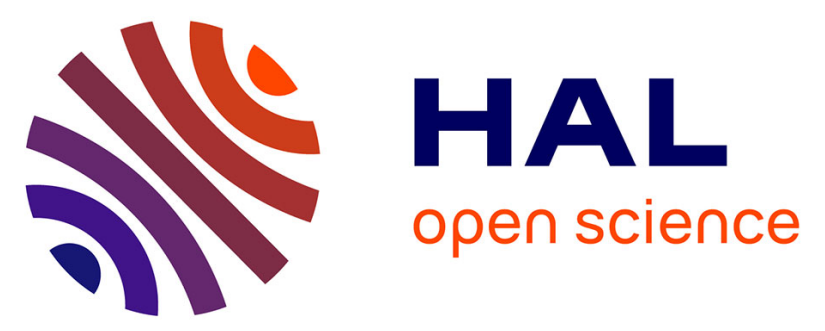

\title{
Highly conductive and transparent coatings from flow-aligned silver nanowires with large electrical and optical anisotropy
}

Ye Xu, Dengteng Ge, Gabriel A Calderon-Ortiz, Annemarie L Exarhos, Coline Bretz, Ahmed M. Alsayed, Dave Kurz, J M Kikkawa, Rémi Dreyfus, Shu Yang, et al.

\section{To cite this version:}

Ye Xu, Dengteng Ge, Gabriel A Calderon-Ortiz, Annemarie L Exarhos, Coline Bretz, et al.. Highly conductive and transparent coatings from flow-aligned silver nanowires with large electrical and optical anisotropy. Nanoscale, 2020, 12 (11), pp.6438-6448. 10.1039/c9nr09598e . hal-02567539

\section{HAL Id: hal-02567539 \\ https://hal.science/hal-02567539}

Submitted on 15 May 2020

HAL is a multi-disciplinary open access archive for the deposit and dissemination of scientific research documents, whether they are published or not. The documents may come from teaching and research institutions in France or abroad, or from public or private research centers.
L'archive ouverte pluridisciplinaire HAL, est destinée au dépôt et à la diffusion de documents scientifiques de niveau recherche, publiés ou non, émanant des établissements d'enseignement et de recherche français ou étrangers, des laboratoires publics ou privés. 


\section{Highly Conductive and Transparent Coatings from Flow-aligned}

\section{Silver Nanowires with Large Electric and Optical Anisotropy ${ }^{\dagger}$}

3 Ye Xu, ${ }^{\neq{ }^{a} a b c}$ Dengteng Ge, ${ }^{\text {fde }}$ Gabriel A. Calderon-Ortiz, ${ }^{b}$ Annemarie L. Exarhos, ${ }^{\text {bs }}$ Coline Bretz, ${ }^{c}$

4 Ahmed Alsayed, ${ }^{\mathrm{c}}$ Dave Kurz, ${ }^{\text {b }}$ J. M. Kikkawa, ${ }^{\mathrm{b}}$ Remi Dreyfus, ${ }^{\mathrm{c}}$ Shu Yang, ${ }^{\mathrm{d}}$ and A. G. Yodh ${ }^{\mathrm{b}}$

5

6 'achool of Mechanical Engineering and Automation and Center of Soft Matter Physics and its

7 Applications, Beihang University, Beijing 100191, P.R. China. Email: ye.xu@buaa.edu.cn

$8 \quad{ }^{b}$ Department of Physics and Astronomy, University of Pennsy/vania, Philadelphia, Pennsy/vania 919104, USA

$10{ }^{c}$ Complex Assemblies of Soft Matter, CNRS-Solvay-UPenn UMI 3254, Bristol, Pennsylvania 19007, USA

$11{ }^{d}$ Department of Materials Science and Engineering, University of Pennsy/vania, Philadelphia,

12 Pennsylvania 19104, USA

13 eState Key Laboratory for Modification of Chemical Fibers and Polymer Materials, Institute of

14 Functional Materials, Donghua University, Shanghai 201620, P.R. China

15

Conductive and transparent coatings consisting of silver nanowires (AgNWs) are promising candidates for emerging flexible electronics applications. Coatings of aligned AgNWs offer unusual electronic and optical anisotropies, with potential for micro-circuits, antennas, and polarization sensors. Here we explore a microfluidics setup and flow-induced alignment mechanisms to create centimeter-scale highly conductive coatings of aligned 
1 AgNWs with order parameters reaching 0.84 , leading to large electrical and optical 2 anisotropy. By varying flow rates, we establish the relation between the shear rate and the 3 alignment and investigate possible alignment mechanisms. The angle-dependent sheet 4 resistance of the aligned AgNW networks exhibits an electronic transport anisotropy of $5 \sim 10 x$ while maintaining low resistivity $(<50 \Omega / s q)$ in all directions. When illuminated, the 6 aligned AgNW coatings exhibit angle- and polarization-dependent color, and the polarized 7 reflection anisotropy can be as large as 25 . This large optical anisotropy is due to a 8 combination of alignment, polarization response, and angle-dependent scattering of the 9 aligned AgNWs.

10

11

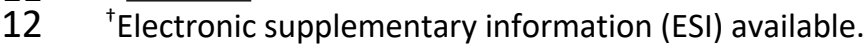

$13{ }^{ \pm}$Authors of equal contributions.

14 SPresent address: Department of Physics, Lafayette College, Easton, PA 18042, USA 


\section{Introduction}

2 The large aspect ratio and electrical conductivity of silver nanowires (AgNWs) offer promising

3 ingredients for emerging electronic and optical applications, including conducting inks and pastes

4 used in circuits. ${ }^{1,2}$ AgNW films, for example, are attractive candidates for transparent conductive

5 coatings in flexible touch-screen displays, due to their high visible light transparency and

6 tolerance to planar strain. ${ }^{3-5}$ Moreover, the one-dimensional (1D) nature of these wires

7 induces anisotropy in electrical conductivity and optical appearance, and some nanowire

8 assemblies with anisotropic properties have been found to exhibit enhanced performance

9 in nanocomputing devices, ${ }^{6}$ antennas, ${ }^{7-9}$ polarized-light-based sensors, ${ }^{10}$ and surface enhanced Raman spectroscopy (SERS). ${ }^{11}$

11 The performance of AgNW-based devices usually depends on the density and organization

12 of the AgNWs. When AgNWs are used in flexible electronics to replace ITO, the combination of a

13 low areal density for optical transparency and a percolated network of AgNWs for conductivity

14 is required. In the literature, this performance is generally achieved from isotropic percolating

15 nanowire networks at a low areal density. $5,12-14$

Films with high areal density of AgNWs, on the other hand, offer the possibility to

17 generate anisotropic optical and electrical properties that ultimately offer many more ways

18 to modulate signals. For example, the fabrication of AgNW-based optical elements with 19 polarization sensitivity and the synthesis of conducting devices with anisotropic conductivity 20 require both a high degree of alignment and a large areal density. In a related vein, the creation

21 of plasmonic waveguides, based on single $e^{15-17}$ or multiple $e^{9,18}$ nanowires, could rely on plasmonic 22 interactions among nanowires ${ }^{19}$, which would benefit from aligned nanowires with high areal 
1 density. To this end, films of aligned nanowires offer the possibility to tune the coupling between

2 individual nanowires and thus introduce novel collective responses, which could be more

3 sensitive than conventional, continuous metal films. Moreover, nanowire films hold potential to

4 be flexible and stretchable, which could be also useful for mechano- and thermal-sensing.

5 Many methodologies for deposition and alignment of 1D nanomaterials such as

6 nanowires or nanotubes have been explored. ${ }^{10,11,20-29}$ Self-assembly at oil-water-air

7 interfaces ${ }^{25}$ and Langmuir-Blodgett methods ${ }^{30}$ have been used to align AgNWs. More

8 recently, capillary-based techniques including capillary printing, ${ }^{31}$ meniscus-dragging, ${ }^{32} \mathrm{H}-$

$9 \mathrm{dip}^{33}$ and bar-coating ${ }^{34}$ have been used to create large-scale aligned or cross-aligned AgNW

10 coatings on flat substrates. Nevertheless, these techniques can only print AgNWs along one

11 specific direction, ${ }^{32,33}$ or require external mechanical force. ${ }^{31}$ Therefore, they are not suitable

12 for parallel printing of complex circuitry. Microfluidic channels, where the shear force and

13 flow geometry can be well-controlled, thus, offering more flexibility for depositing AgNW

14 coatings with pre-designed patterns. Indeed, shear flow has been used for aligning

15 nanotubes and nanowires. Huang et al., first explored this method to align nanowires within

16 a microfluidics apparatus. ${ }^{35}$ They created nanoscale network structures with a very low areal

17 density. Later, Liu et al. employed shear flow in circular glass capillaries to prepare more

18 dense and aligned AgNW coatings. ${ }^{11}$ Though very efficient, because of geometric constraints,

19 these nanowires cover only the interior of circular glass tubes. Clearly, there remains a need

20 for new methods to assemble AgNWs into scalable and aligned structures of high areal

21 density with adequate light transmission, to achieve electrical and optical anisotropy for

22 applications such as polarization light sensors and plasmonic waveguides. 
In this contribution, we employ flow-assisted assembly to generate centimeter-scale

2 AgNW coatings at high areal density (approximately $40 \%$ areal coverage or $10^{4}$

3 nanowires $\left./ \mathrm{mm}^{2}\right)$, with high conductivity $(<10 \Omega / \mathrm{sq})$, and with controlled electric and optical

4 anisotropy on planar surfaces. The robust method enables experimental control of

5 alignment via variation of the shear rate of the AgNW suspension near the substrate surface.

6 We systematically investigate the angular dependence of coating sheet resistance and

7 optical properties. These properties are strongly affected by AgNW alignment. The

8 anisotropy of electric conductivity is $\sim 10 x$ in well-aligned AgNW coatings. Moreover, by

9 combining polarized illumination and detection of scattered light from the aligned AgNW

10 coatings, the degree of polarized reflection anisotropy can reach up to $\sim 25 x$. The films also

11 have good transparency ( $70 \%)$ in the normal incidence across the visible spectrum. The

12 performance and features of AgNW films based on our deposition technique, along with

13 those from previous literature, are reported in Table 1. Our transparent coatings in the

14 planar geometry have comparatively high areal density and larger electrical and optical

15 anisotropy due to better control of AgNW orientation and concentration. These features

16 make them attractive for components in micro-/nano-circuits and optical sensors.

18 Results and discussions

Preparation of AgNW coatings

Thin coatings of AgNWs are prepared by flowing nanowire suspensions through thin

21 rectangular microfluidic channels, as shown in Fig. 1A. At the beginning of the coating

22 preparation, we utilize a syringe pump (Harvard Instrument) to drive the flow of $2 \mathrm{~mL}$ of 
nanowire suspension through the channel at a constant volumetric flow rate, $Q$, ranging

2 from 0.5 to $5 \mathrm{~mL} / \mathrm{min}$. The flow of AgNW suspension creates boundary shear at a rate

3 ranging from $10 s^{-1}$ to $1000 s^{-1}$ near the top and bottom glass walls of the channel,

4 respectively. As a result, a fraction of the nanowires are observed to deposit onto the glass

5 slide surface that is exposed to the AgNW suspension. Subsequently, $1 \mathrm{~mL}$ pure ethanol is

6 flowed through the channel to remove excess nanowire suspension, followed by a gas

7 purge and drying to remove liquid remaining inside the channel. AgNWs deposited on the

8 top and bottom walls of the channel are not washed out with the main flow because the

9 Van der Waal forces are of order $10^{-6} \mathrm{~N}^{36}$ between the nanowire and the glass slide and are

10 therefore strong enough to prevent the shear flow forces (of order $10^{-9} \mathrm{~N}$ ) from displacing

11 the nanowire. At the end of the preparation process, a uniform thin layer of AgNWs forms

12 at the surface of each glass slide as shown in Fig. 1B. The coated glass slides are then

13 separated for further characterization.

14 As shown in Fig. 1C-E micrographs, nanowires coated on the surface of the glass slides

15 exhibit increased alignment with increasing suspension flow rate through the channel. The

16 deposited nanowires are poorly aligned at low flow rates, i.e. $0.5 \mathrm{~mL} / \mathrm{min}$, as shown in Fig.

17 1C-i. With increasing flow rate, i.e. to $2 \mathrm{~mL} / \mathrm{min}$ and $5 \mathrm{~mL} / \mathrm{min}$, the deposited nanowires

18 begin to align along the flow direction over the whole area inside the microchannels (Fig.

19 1C-ii and iii). While aspects of this flow alignment effect have been explored and utilized in

20 previous studies, ${ }^{11,20,35,37,38}$ the present experimental methods provide a simple and robust

21 way to generate centimeter-scale and potentially patternable AgNW coatings on a flat

22 substrate with high areal density and controlled alignment. 


\section{Alignment of AgNWs}

We characterize the microscopic structure of nanowire networks by analyzing the

4 micrographs obtained by both optical and scanning electron microscopy (SEM). Examples

5 of micrographs are shown in Fig. 2A-C. They are taken from samples prepared at three

6 different suspension flow rates: $0.5,2$, and $5 \mathrm{~mL} / \mathrm{min}$, respectively. For analysis, each

7 individual nanowire in the field-of-view is approximated by a straight-line segment. The

8 orientation, $\vartheta$, and length, $I$, of the AgNW are then recorded by image processing using

9 software ImageJ. Here we define $\theta=0^{\circ}$ as the direction of the flow.

The distributions of the nanowire orientation are shown in the histograms of Fig. 2D.

11 For the image in Fig. 2A, the probability distribution is very broad and flat, suggesting a

12 predominately random distribution of the nanowire orientation. By contrast, the

13 probability distribution of nanowire orientation from images in Fig. $2 \mathrm{~B}$ and $2 \mathrm{C}$ exhibits a

14 clear peak near $\vartheta=0^{\circ}$. The presence of these peaks clearly demonstrates that the

15 orientations of deposited AgNWs are biased toward the flow direction. These different

16 distributions of nanowire orientation are also consistent with the intensity patterns

17 generated by laser light scattering. As shown in the insets of Fig. 2E, the AgNW coating with random distribution of nanowire orientation exhibits a nearly isotropic scattering pattern

19 in the $k$-space, while the one with aligned AgNWs exhibits a strongly anisotropic scattering 20 pattern.

21 We further quantify alignment by the two-dimensional nematic order parameter defined $22 \mathrm{as}^{39,40}$ 


$$
S=2<\cos ^{2} \theta>-1
$$

2 where $S=0$ corresponds to a random distribution, and $S=1$ corresponds to perfect

3 alignment. Using the distribution of $\theta$ in Fig. 2D, the three samples shown in Fig. 2A-C give $4 S$ values of $0.16,0.61$, and 0.84 , respectively.

5 The shear rate near the glass surfaces plays an important role in controlling the degree 6 of alignment of AgNWs in these flow-deposited coatings. Given the density, $\rho$, viscosity, $\mu$, 7 and flow rate, $\mathrm{u}$, of the AgNW suspension, together with the geometry of the channel 8 (height, $\mathrm{H}$, and width, $\mathrm{W}$ ), the Reynolds' number, $\mathrm{Re}=\rho \mathrm{H} / \eta$, is of order $10^{-2}$. This 9 indicates that flow is laminar. Therefore, assuming no-slip boundary conditions, for a 10 volumetric flow rate, $\mathrm{Q}$, the shear rate near the wall can be calculated as $\gamma=6 \mathrm{Q} /\left(\mathrm{H}^{2} \mathrm{~W}\right)$. 11 In Fig. 2E, we plot the nematic alignment order parameter of the deposited AgNW coatings 12 deposited versus shear rates. The suspended nanowires that have made point-contact with 13 the glass surface respond to the high shear rate near the surface, introduced by the rapid channel flow, by adopting a preferred orientation parallel to the flow.

To better understand the flow-induced alignment behaviors, we perform semi-quantitative analyses on experimental observations. We first rule out gravity or sedimentation effects, since AgNWs adhere equally to both top and bottom surfaces of the thin channel. Therefore, a possible explanation for the effect of flow-induced alignment could derive from a competition between directional shear flow and random rotational diffusion of the AgNWs. At a low shear rate, the rotational diffusion dominates and gives rise to a more isotropic organization of the

$21 \mathrm{AgNWs}$, but at large shear rates the flow defines the orientation. We then estimate the Peclet 22 number $(P e)$ for the AgNW suspension, defined by the ratio between the shear rate and the 
1 rotational diffusion constant of the nanowire, $D_{r}$, as ${ }^{33,41} \mathrm{Pe}=\dot{\gamma} / D_{r}=\pi \dot{\gamma} \mu L^{3} / 4 k_{B} T$, where

$2 \mu \approx 1.2 \mathrm{mPa} \cdot \mathrm{s}$ is the viscosity of the AgNW suspension and $L \approx 30 \mu \mathrm{m}$ is the average length

3 of AgNWs. For the range of experimental shear rates, $\dot{\gamma}=10$ to $1000 \mathrm{~s}^{-1}, P e$ is of the order of

$410^{6}$ to $10^{8}$. The large $P e$ imply that shear flow should dominate over rotational diffusion in all

5 the experimental flow conditions used for preparing AgNW coatings. We believe that the key to

6 the alignment of nanowires on the glass surface occurs when the rods make contact with the

7 surface (i.e. rather than in the bulk fluid). Just after making contact, and assuming this contact

8 occurs at one end of the nanowire with the other end free, the nanowires can pivot and will

9 respond to flow shear-stresses near the surface. Here, the most plausible mechanism is a competition between electrostatic forces that pull the nanowire to the glass substrate and the

11 hydrodynamic drag force of the shear flow that rotates the AgNWs along a direction parallel to

12 the flow when they get contact with the glass surface. Here, by assuming a line charge density

$13 \delta$ for the nanowire and a surface charge density $\sigma$ for the glass surface, we can estimate the

14 ratio between electrostatic torque $\left(M_{\text {elec }}\right)$ and hydrodynamic torque $\left(M_{\text {hydro }}\right)$,

$$
\frac{M_{\text {hydro }}}{M_{\text {elec }}} \sim \frac{4}{3} \frac{\gamma \eta L \epsilon_{0} \epsilon_{r}}{\delta \sigma}
$$

16 where $\epsilon_{0} \epsilon_{r}$ is the electrical permittivity of the AgNW suspension. The detailed illustration of

17 those two torques can be found in the ESI. With the increasing shear rate, $\gamma$, it is possible to transition from an electrostatic-dominated regime to a shear-flow-dominated regime. In the

19 former regime, AgNWs are pulled down to the glass surface with random orientations before 20 they can be aligned by flow; in the latter region, AgNWs are quickly aligned by the high shear

21 rate near the surface. In our experiments, given the glass surface charge on the order of

$221 \mathrm{mC} / \mathrm{m}^{2}$, and a transition of shear rate at approximately $\gamma=100 \mathrm{~s}^{-1}$, according to Eq. 2, the 
1 nanowires carry a line charge density on the order of $3 \times 10^{-12} \mathrm{C} / \mathrm{m}$, or 20 effective charges

2 per micron. Although further experimental confirmation of these predictions is difficult, the

3 estimation is plausible for the observed transition of the AgNW orientation as a function of the

4 shear rate near the surface.

5

6 Electrical conductivity and anisotropy

7 One advantage of our system versus literature ${ }^{11}$ is that we can break apart the 8 microchannel to characterize the electrical resistance of the AgNW coatings on the surface

9 of glass slides. To explore the angle-dependence of surface conductivity in our AgNWs coatings, we use a four-point probe setup and a rotating stage to measure the sheet

11 resistance, $R_{s}$, at various angles, $\theta$, relative to the flow direction at the same location. The

12 sheet resistances, $R_{s}$, of three AgNW coatings with different degrees of alignment are

13 plotted in Fig. 3A as a function of $\theta$. For samples with good alignment, we find significant

14 differences in measured sheet resistance at different angles. At the angle parallel to the flow

15 direction, i.e. $\theta=0^{\circ}$ and $180^{\circ}$, the measured $R_{s}$ are in the range of 3-5 $\Omega /$ sq. However, at $\vartheta$

$16=90^{\circ}$ and $270^{\circ}, R_{s}$ increases to $\sim 42 \Omega / \mathrm{sq}$, i.e., an order of magnitude higher. Accordingly,

17 we extract the angle-dependent sheet resistance of these aligned samples as:

18

$$
R_{s, \theta}=a \sin (\theta)+R_{0}
$$

19 Here $a$ and $R_{0}$ are fitting parameters. We find a good agreement between the data and the

20 sinusoidal waveform. By contrast, the sheet resistance for an AgNW coating with random

21 orientations show no angle dependence, $\sim 3 \Omega /$ sq. 
To further quantify the effect of alignment, we define the electrical anisotropy, $\sigma_{a n i}$,

2 as the ratio between maximum and minimum sheet resistance measured at different angles,

3 i.e. $\sigma_{a n i}=R_{s, \max } / R_{s, \min }$, and we plot the ratio as a function of the AgNW coating order

4 parameter, $S$ (Fig. 3B). A strong anisotropy, $\sigma_{\text {ani }}$ up to $\sim 10$ is reached for highly aligned

5 AgNW coatings $(S=0.84)$. We note that alignment-induced electrical anisotropy has also

6 been reported in coatings of carbon nanotubes (CNTs). ${ }^{40}$ Our observations about the change

7 of anisotropy with degree of alignment, $S$, are roughly consistent with the results measured

8 for the CNT coatings, which is well-described by a model assuming a Gaussian distribution of

9 the orientations of 1D conducting elements on a planar substrate. Anisotropy of electrical

conductivity in aligned AgNW coatings has been reported. Ackermann et al. ${ }^{42}$ reported $\sigma_{\text {ani }}$

11 varying from 1.06 to 27.6. However, high electrical anisotropy, i.e. $\sigma_{a n i}>2$, was observed

12 only in samples with sheet resistance larger than $100 \Omega / s q$. To our knowledge, the well-

13 aligned AgNWs used in the present work are the first to exhibit a large anisotropy ( 10) while

14 maintaining low sheet resistance $(<50 \Omega / \mathrm{sq})$ in all directions.

\section{Optical anisotropy}

Interestingly, the anisotropy of the coatings has a direct impact on their optical properties. We characterize the optical response of coatings made of aligned AgNW using the setup shown in Fig. 4A. In this setup, the sample is illuminated with a Tungsten halogen light source (HL-2000, Ocean Optics) at normal incidence angle (along - $z$ direction), and the

21 AgNW coatings are placed in the $x y$-plane. The camera or detector is placed in the $x z$-plane 22 with the polar angle, $\phi$, measured relative to the $z$-axis. The angle between the polarization 
1 of the incident light and the $y$-axis is defined as $\alpha$. For aligned AgNW coatings, the angle

2 between the nanowire alignment direction and the $y$-axis is defined as $\beta$.

The images in Fig. 4B show the optical images of a well-aligned AgNW coating $(\mathrm{S}=0.84)$

with light polarized along the $y$-axis $\left(\alpha=0^{\circ}\right)$ and $x$-axis $\left(\alpha=90^{\circ}\right)$, recorded at $\phi=60^{\circ}$. The sample appearance varies from orange to yellow-green when $\alpha$ changes from $0^{\circ}$ to $90^{\circ}$. In

6 addition, a sharp decrease of the scattered light intensity occurs when $\beta$ is rotated from

$70^{\circ}$ to $90^{\circ}$, for both $\alpha=0^{\circ}$ and $\alpha=90^{\circ}$. By contrast, the color and intensity from the

8 coatings of poorly aligned AgNWs remain the same (Fig.5A, B)

We further investigate the color-changing mechanism by taking micrographs of the AgNW coatings using a microscope with a polarized light source. As shown in insets in Fig.

$114 \mathrm{C}$, under the reflection mode, i.e. $\phi=0^{\circ}$, and when the polarization and the AgNWs are

12 aligned $\left(\alpha=0^{\circ}\right.$ and $\left.\beta=0^{\circ}\right)$, the sample is mostly yellow. By contrast, the sample appears

13 blue-green when the polarizer is perpendicular to the $\mathrm{AgNW}$ alignment $\left(\alpha=90^{\circ}\right.$ and $\beta=$

$\left.0^{\circ}\right)$. In the transmission mode $\left(\phi=180^{\circ}\right)$, the trends are opposite, as shown in insets in Fig.

15
4D.

To more quantitatively explain the color differences, the reflectance and transmittance spectra are recorded. As shown in Fig. 4C, when $\alpha=0^{\circ}$, the intensity of reflected light increases as a function of wavelength between $420 \mathrm{~nm}$ and $800 \mathrm{~nm}$. When $\alpha=90^{\circ}$, however, the reflected intensity decreases. These findings are consistent with the colors observed from the optical microscope (insets in Fig. 4C).

The transmittance spectra recorded by the UV-Vis spectrometer are plotted in Fig. 4D. While we did not optimize for transparency, the films retained good light transmission 
1 across the visible spectral range ( $70 \%)$. The aligned AgNW coating exhibit absorbing

2 valleys for illumination in both polarization directions. However, these two valleys are

3 centered about two very different wavelengths. For $\alpha=0^{\circ}$ and $\beta=0^{\circ}$, a strong

4 absorption peak arises at long wavelengths, within the range of $600-650 \mathrm{~nm}$, as shown in

5 Fig $4 \mathrm{D}$. For $\alpha=90^{\circ}$ and $\beta=0^{\circ}$, the peak absorption arises at much shorter wavelengths,

6 i.e., $\sim 380 \mathrm{~nm}$. These spectra exhibit features similar to those of spectra reported for AgNWs

7 in solution ${ }^{43}$ and aligned AgNW bundles. ${ }^{30,33}$ Prior simulation ${ }^{44}$ and experimental ${ }^{11,33,45}$

8 work explains the absorption peak at short (long) wavelength as due to the transverse

9 (longitudinal) surface plasmon resonance (SPR) of the electrons in the AgNWs ${ }^{30}$. Specifically,

10 the absorption peak at short wavelength is due to the transverse surface plasmonic

11 resonances (SPRs) induced by electron oscillation along the AgNW diameter, and the

12 absorption at the long wavelength is due to the longitudinal SPRs (induced parallel to the

13 AgNW long axis). The broadening of the peaks could be due, in part, to the polydispersity

14 in the AgNW distribution and to the coupling of electromagnetic waves among neighboring

15 nanowires.

When stimulated by polarized light, the wavelength of the absorption peak depends

17 on the angle between the individual nanowire and the light polarization. This angle-

18 dependent absorption is clearly seen in micrographs of randomly oriented AgNWs in Fig.

19 5E and $\mathbf{5 F}$, where an individual AgNW imaged in transmission appears yellow or green

20 depending on its orientation relative to the light polarization. However, because of the

21 random orientation of AgNWs, the overall spectra of the whole AgNW coating does not

22 show absorption valleys, as seen in Fig. 5C and 5D. By contrast, when AgNWs are aligned 
1 along the same direction, the SPR absorption spectra of the aligned individual AgNWs are

2 approximately the same and the sample as a whole exhibits a clear valley in its transmission

3 spectra, as shown in Fig 4D and 4E.

$4 \quad$ We systematically quantify the angle-dependent light intensity under various

5 illumination and observation configurations to fully explore the optical anisotropy of the

6 strongly aligned AgNW coating $(S=0.84)$ at $650 \mathrm{~nm}$. The polar plot in Fig. 6 A shows the

7 intensity, I, of the reflected light, i.e. $\phi=0^{\circ}$, as a function of the angle between the

8 input polarizer and the AgNW alignment direction. The plot in Fig. 6A clearly

9 demonstrates optical anisotropy; however, the value, $\frac{I_{\max }}{I_{\min }}=1.7$, is rather small and

10 the normalized light intensity peak at $180^{\circ}$ is quite broad with Full-Width-at-Half-

11 Maximum (FWHM) of approximately $92^{\circ}$. This result is consistent with previous studies

12 where weak optical anisotropy $(<2 \mathrm{x})$ is observed from individual aligned nanotubes and

13 from AgNWs in both transmission and reflection modes. ${ }^{10,11,46}$

14 Next, we illuminate the aligned AgNW coating with unpolarized light and measure

15 the intensity of scattered light at $\phi=60^{\circ}$; for this measurement, the angle of aligned

16 AgNW coating, $\beta$, is varied by rotating the sample. The results are shown in Fig. $6 \mathrm{~B}$, which

17 exhibits a large anisotropy, $\frac{I_{\max }}{I_{\min }}=7.2$, and a narrow peak of normalized intensity with

18 FWHM of approximately $24^{\circ}$. For comparison, the intensity of unpolarized light

19 scattered by a coating with randomly oriented AgNWs shows no variation as a function

20 of $\beta$. These results indicate that the coating with aligned AgNWs scatters more light in

21 the direction perpendicular to the AgNW alignment direction, and less light in the 
1 direction parallel to the alignment direction. This type of effect is observed in optical

2 gratings with parallel stripe structure on the surface. ${ }^{47} \mathrm{It}$ is also worth noting that the

3 aligned AgNW coating scatters less light in total when integrating over $\beta$, compared to

4 a randomly oriented AgNW coating. This scattering could potentially potential affect

5 the haze of the coating. Reduction of haze by alignment has been studied previously ${ }^{31}$

6 and is expected to decrease with better alignment, but we leave quantification of haze

7 in our samples for future work.

8 Finally, we combine the two anisotropic effects by measuring the intensity of light 9 scattered from polarized light source. Specifically, for $\alpha=0^{\circ}$, and the intensity of scattered light at a constant azimuthal $\phi=60^{\circ}$ is measured as a function of different

11 angles $\beta$. As shown in Fig. 6C, this configuration produces a very large optical anisotropy,

12 with $\frac{I_{\max }}{I_{\min }}=25.6$, and a very narrow peak, with FWHM of approximately $15^{\circ}$. The comparison of normalized light intensity versus $\beta$ for various values of $\alpha$ is shown in Fig.

$146 \mathrm{D}$. Clearly, the directional alignment of $\mathrm{AgNWs}$ and the polarization optical absorption 15 and scattering properties of the individual AgNWs contribute to overall optical anisotropy of the well-aligned AgNW coating. When the two mechanisms are combined, we observe a much higher anisotropy than reported in earlier studies. ${ }^{10,11,46}$

Large optical anisotropy has been shown to improve the resolution of signals such as those arise in polarization images or surface-enhanced Raman spectroscopy (SERS), ${ }^{30}$ and large anisotropy is desirable in nanowire-based polarizers for polarization light sensors.

21 Normally, researchers can use the different responses of aligned metal nanowires under 22 different polarization light conditions to achieve the optical anisotropy. Previous studies 
1 showed that transmission or reflection modes typically give an optical anisotropy of less

2 than $2 x^{11,25}$ in agreement with the results shown in Fig. 6 A. Here, we have uncovered a

3 novel way to further increase the optical anisotropy that relies on the light scattered by the

4 aligned AgNWs. This detection configuration, wherein well-aligned AgNW coatings are

5 illuminated with polarized light and the light scattered by the coating is detected at

6 different polar angles, takes advantage of both the intrinsic polarization properties of

$7 \quad$ AgNWs and the angle-dependent scattering of aligned the AgNWs to achieve greater optical

8 anisotropy, which can be as large as $25 \mathrm{x}$ as demonstrated in Fig. 6C.

10 Conclusions

11 We developed a simple and robust flow-induced alignment method for preparing

12 centimeter-scale planar conductive silver nanowire coatings with high degrees of anisotropy.

13 Specifically, we demonstrated patterning of a $5 \mathrm{~mm}$ by $2 \mathrm{~cm}$ film strip of AgNWs with an

14 orientational order parameter that was tunable from 0.16 to 0.84 and had concomitant

15 variation in electrical and optical anisotropy. The alignment can be tuned by varying the shear

16 rate of nanowire suspension near the substrate during the deposition process. As a result of the

17 preferred direction of AgNWs, coatings consisting of highly aligned AgNWs exhibit large

18 anisotropy in both electrical conductivity and optical properties. For electrical conductivity, an

19 anisotropy of $\sim 10 \mathrm{x}$ is achieved, while the overall sheet resistance remains low. A very large

20 optical anisotropy of $\sim 25 \mathrm{x}$ is observed in the scattered polarized light due to the combination of

21 the intrinsic polarization property of individual AgNWs and "grating" effects of the aligned

22 AgNWs network. Those highly conductive transparent coatings with large electrical and 
1 optical anisotropy can be potentially used for design of novel flexible antennas, polarized-

2 light-based sensors, and for surface enhanced Raman spectroscopy.

\section{Experimental section}

5 Silver Nanowire Suspension. The silver nanowires (AgNWs) were purchased from

6 BlueNano (SLV-NW-90). The average length and diameter of the nanowires are $30 \pm 5 \mu \mathrm{m}$

7 and $90 \pm 20 \mathrm{~nm}$, respectively. The nanowires are suspended in ethanol at a solid 8 concentration of $\sim 1$ wt\%. The nanowire suspension was used as-received.

9

Assembly of The Microfluidic Channel. To fabricate the channels, two glass slides are attached (sandwiched) using two strips of double-sided tape, leaving a thin gap, as shown in Fig. 1A. The resulting rectangular channels are typically $25 \mathrm{~mm}$ in length, $5 \mathrm{~mm}$ in width, and 200 to $600 \mu \mathrm{m}$ in height. The two open ends of the channel are then connected to a syringe filled with AgNW suspension and a waste bottle.

Electrical Characterization. The sheet resistances of the resulting AgNW coatings are measured using a four-point probe setup to eliminate the effect of contact resistance between the electrodes and coatings. Four electrical probes made of thin gold wires are arranged to be parallel and equally spaced. The part of the probe that contacts the nanowire coating has length $w=2 \mathrm{~mm}$, and each probe is separated by $d=0.5 \mathrm{~mm}$, as shown in Fig. S3 in ESI. A constant DC current, I, is applied through the two outmost probes, and the voltage, $U$, is measured between the two inner probes. The sheet resistance, $R_{s}$, is 
then calculated by

$$
R_{s}=\frac{U}{I} \frac{w}{d}
$$

3 where $R_{s}$ has a unit of $\Omega /$ sq. This setup permits measurement of the intrinsic electrical

4 conductivity of the nanowire coatings. To explore the angle-dependence of surface

5 conductivity of our AgNWs coatings, we place the coatings on a rotating stage wherein $R_{s}$

6 can be measured at various angles relative to the flow direction at the same location.

8 Optical characterization. The micrographs of AgNW samples are recorded using an optical 9 microscope (Olympus BX61) in both transmission and reflectance modes. In addition, the 10 reflectance and transmittance spectra are recorded by coupling, respectively, a fiber 11 spectrometer (USB4000, Ocean Optics) and a UV-Vis spectrometer (Cary 5000, Agilent 12 Technologies) to the Olympus microscope.

\section{Acknowledgements}

15 We thank Prof. Karen Winey for helpful discussions. The project is supported by the National 16 Science Foundation through DMR16-07378, MRSEC DMR-1720530, including both its Optical 17 Microscopy and its Properties Measurement Shared Experimental Facilities, MRSEC DMR1118 20901, and NASA NNX08AO0G. This work is also supported in part by Fundamental Research

19 Funds for the Central Universities, the National Natural Science Foundation of China through 20 Grants No. 11674019 (Y.X.) and 11604045 (D.G.), Projects from Shanghai Science \& Technology 21 Commission 17JC1400700 and 17ZR1440000, and Shanghai Pujiang Program 16PJ1400100 (D. 22 G.). 


\section{Author Contributions}

3 Y.X., D.G., and A.G.Y. conceived and designed the project; Y.X., G.A.C., A.A., and D.K. carried out

4 experiments for sample preparation; Y.X., A.L.E., and J.M.K. carried out electric characterization

5 experiments; D.G. and C.B. carried out optical characterization experiments; Y.X., D.G., R.D., and

6 A.G.Y. analyzed the data and wrote the manuscript. All authors discussed the results and 7 commented on the manuscript.

8

\section{References}

11 1. D. J. Finn, M. Lotya and J. N. Coleman, ACS Applied Materials \& Interfaces, 2015, 7, 9254129261.

13 2. J. Liang, K. Tong and Q. Pei, Advanced Materials, 2016, 28, 5986-5996.

14 3. E. M. Freer, O. Grachev, X. Duan, S. Martin and D. P. Stumbo, Nat. Nanotechnol., 2010, 5, $15 \quad 525-530$.

16 4. F. Xu and Y. Zhu, Adv. Mater., 2012, 24, 5117-5122-5117-5122.

17 5. L. Hu, H. S. Kim, J. Y. Lee, P. Peumans and Y. Cui, ACS Nano, 2010, DOI: 10.1021/nn1005232.

18 6. J. Yao, H. Yan, S. Das, J. F. Klemic, J. C. Ellenbogen and C. M. Lieber, Proceedings of the $19 \quad$ National Academy of Sciences, 2014, DOI: 10.1073/pnas.1323818111.

20 7. D. Rossouw, M. Couillard, J. Vickery, E. Kumacheva and G. A. Botton, Nano Letters, 2011, $21 \quad 11,1499-1504$.

22 8. L. Song, A. C. Myers, J. J. Adams and Y. Zhu, ACS Applied Materials \& Interfaces, 2014, 6, $23 \quad 4248-4253$.

$249 . \quad$ L. Lu, L.-L. Wang, C.-L. Zou, X.-F. Ren, C.-H. Dong, F.-W. Sun, S.-H. Yu and G.-C. Guo, The 25 Journal of Physical Chemistry C, 2012, 116, 23779-23784.

26 10. X. Ma, X. Zhu, F. You, J. Feng, M.-C. Wang and X. Zhao, Journal of Alloys and Compounds, 
2014, 592, 57-62.

11. J. W. Liu, J. L. Wang, W. R. Huang, L. Yu, X. F. Ren, W. C. Wen and S. H. Yu, Sci Rep, 2012, 2, 987.

12. R. Mutiso and K. Winey, Phys. Rev. E: Stat., Nonlinear, Soft Matter Phys., 2013, 88.

13. R. M. Mutiso, M. C. Sherrott, A. R. Rathmell, B. J. Wiley and K. I. Winey, ACS nano, 2013.

14. S. White, B. DiDonna, M. Mu, Lubensky and K. Winey, Phys. Rev. B, 2009, 79.

15. D. Zhang, Y. Xiang, J. Chen, J. Cheng, L. Zhu, R. Wang, G. Zou, P. Wang, H. Ming, M. Rosenfeld, R. Badugu and J. R. Lakowicz, Nano letters, 2018, 18, 1152-1158.

16. A. W. Sanders, D. A. Routenberg, B. J. Wiley, Y. Xia, E. R. Dufresne and M. A. Reed, Nano Letters, 2006, 6, 1822-1826.

17. W. Wang, Q. Yang, F. Fan, H. Xu and Z. L. Wang, Nano letters, 2011, 11, 1603-1608.

18. X. Guo, M. Qiu, J. Bao, B. J. Wiley, Q. Yang, X. Zhang, Y. Ma, H. Yu and L. Tong, Nano Letters, 2009, 9, 4515-4519.

19. D. Lei, A. Aubry, Y. Luo, S. A. Maier and J. B. Pendry, ACS Nano, 2011, 5, 597-607.

20. S.-k. Duan, Q.-I. Niu, J.-f. Wei, J.-b. He, Y.-a. Yin and Y. Zhang, Phys. Chem. Chem. Phys., 2015, 17, 8106-8112.

21. Y. Jeon, H. Kang, S. Ko and H. Sung, Measurement Science and Technology, 2013, DOI: 10.1088/0957-0233/24/3/035303.

22. Y. Li and Y. Wu, Journal of the American Chemical Society, 2009, DOI: 10.1021/ja9000882.

23. Y. Liu, J.-H. Chung, W. Liu and R. S. Ruoff, The Journal of Physical Chemistry B, 2006, DOI: 10.1021/jp061367e.

24. S. J. Papadakis, Z. Gu and D. H. Gracias, Appl. Phys. Lett., 2006.

25. H.-Y. Shi, B. Hu, X.-C. Yu, R.-L. Zhao, X.-F. Ren, S.-L. Liu, J.-W. Liu, M. Feng, A.-W. Xu and S.H. Yu, Advanced Functional Materials, 2010, 20, 958-964.

26. W. Yang, L. Qu, R. Zheng, Z. Liu, K. R. Ratinac, L. Shen, D. Yu, L. Yang, C. J. Barrow, S. P. Ringer, L. Dai and F. Braet, Chemistry of Materials, 2011, DOI: 10.1021/cm1033645.

27. R. Zhu, Y. Lai, V. Nguyen and R. Yang, Nanoscale, 2014, DOI: 10.1039/C4NR02645D.

28. L. Meng, R. Bian, C. Guo, B. Xu, H. Liu and L. Jiang, Advanced Materials, 2018, 30, 1706938.

29. C. D. Preston, L. Hu and L. J. Martínez-Miranda, Molecular Crystals and Liquid Crystals, 
2015, 610, 235-239.

30. A. Tao, F. Kim, C. Hess, J. Goldberger, R. He, Y. Sun, Y. Xia and P. Yang, Nano Lett., 2003, 3, 1229-1233.

31. S. Kang, T. Kim, S. Cho, Y. Lee, A. Choe, B. Walker, S. J. Ko, J. Y. Kim and H. Ko, Nano Lett, $2015,15,7933-7942$.

32. Y. Ko, S. K. Song, N. H. Kim and S. T. Chang, Langmuir, 2016, 32, 366-373.

33. B. Park, I. G. Bae and Y. H. Huh, Sci Rep, 2016, 6, 19485.

34. S. Cho, S. Kang, A. Pandya, R. Shanker, Z. Khan, Y. Lee, J. Park, S. L. Craig and H. Ko, ACS Nano, 2017, 11, 4346-4357.

35. Y. Huang, X. Duan, Q. Wei and C. M. Lieber, Science, 2001, 291, 630-633.

36. F. L. Leite, C. C. Bueno, A. L. Da Roz, E. C. Ziemath and O. N. Oliveira, Int J Mol Sci, 2012, 13, $12773-12856$.

37. S. B. Kharchenko, J. F. Douglas, J. Obrzut, E. A. Grulke and K. B. Migler, Nature Materials, 2004, DOI: 10.1038/nmat1183.

38. M. Liu, Y. Chen, Q. Guo, R. Li, X. Sun and J. Yang, Nanotechnology, 2011.

39. P. J. Steinhardt, D. R. Nelson and M. Ronchetti, Physical Review B, 1983, 28, 784-805.

40. C. Zamora-Ledezma, C. Blanc, N. Puech, M. Maugey, C. Zakri, E. Anglaret and P. Poulin, Physical Review E, 2011, 84, 62701.

41. M. Doi and S. F. Edwards, The theory of polymer dynamics, oxford university press, 1988.

42. T. Ackermann, R. Neuhaus and S. Roth, Sci Rep, 2016, 6, 34289.

43. Q. N. Luu, J. M. Doorn, M. T. Berry, C. Jiang, C. Lin and S. P. May, Journal of colloid and interface science, 2011, 356, 151-158.

44. J. Dong and I. A. Goldthorpe, Nanotechnology, 2017, 29, 45705.

45. B. Pietrobon, M. McEachran and V. Kitaev, ACS Nano, 2009, 3, 21-26.

46. M. F. Islam, D. E. Milkie, C. L. Kane, A. G. Yodh and J. M. Kikkawa, Physical Review Letters, $2004,93,37404$.

47. G. J. Dunning and M. L. Minden, Applied Optics, 1980, 19, 2419. 
1 Table 1: Comparison of electrical and optical properties of AgNW films prepared with different aligning methods

\begin{tabular}{|c|c|c|c|c|c|c|c|c|}
\hline $\begin{array}{c}\text { Sample } \\
\text { preparation }\end{array}$ & $\begin{array}{c}\text { Alignment } \\
\text { quantified } \\
?\end{array}$ & $\begin{array}{c}\text { Areal } \\
\text { density/ } \\
\text { fraction }\end{array}$ & $\begin{array}{l}\text { Sheet } \\
\text { resistance }\end{array}$ & $\begin{array}{c}\text { Electrical } \\
\text { anisotropy }\end{array}$ & $\begin{array}{l}\text { Transp } \\
\text { arency }\end{array}$ & $\begin{array}{c}\text { Linear } \\
\text { Optical } \\
\text { Anisotropy }\end{array}$ & $\begin{array}{l}\text { Patter } \\
\text { ning? }\end{array}$ & Scale \\
\hline $\begin{array}{c}\text { Self- } \\
\text { assembly at } \\
\text { water-oil } \\
\text { interface }^{25}\end{array}$ & $\mathrm{~N}$ & $\begin{array}{l}\text { Close- } \\
\text { packed }\end{array}$ & NM & NM & NQ & NQ & $\mathrm{N}$ & $10 \mathrm{~mm}$ \\
\hline $\begin{array}{l}\text { Langmuir-BI } \\
\text { odgett }^{30}\end{array}$ & $\mathrm{~N}$ & $\begin{array}{l}\text { Close- } \\
\text { packed }\end{array}$ & NM & NM & $\mathrm{NQ}[\mathrm{a}]$ & $\mathrm{NQ}$ & $\mathrm{N}$ & $50 \mathrm{~mm}$ \\
\hline $\begin{array}{l}\text { Flow } \\
\text { through } \\
\text { circular } \\
\text { capillary } \\
\text { tube }{ }^{11}\end{array}$ & $\mathrm{~N}$ & $N Q[b]$ & NM & NM & $\mathrm{NQ}$ & $\sim 2$ & $\mathrm{~N}$ & $\begin{array}{c}300 \mu \mathrm{m}[\mathrm{c} \\
]\end{array}$ \\
\hline $\begin{array}{l}\text { Capillary } \\
\text { printing }^{31}\end{array}$ & $Y$ & $0.3-20 \%$ & $10-50 \Omega / s q$ & $\sim 1.7$ & $90-95 \%$ & NM & $\mathrm{N}$ & $\sim 10 \mathrm{~mm}$ \\
\hline $\begin{array}{l}\text { Meniscus- } \\
\text { dragging }^{32}\end{array}$ & $\mathrm{~N}$ & $\begin{array}{c}3- \\
12 \times 10^{4} \\
\mathrm{NW} / \mathrm{m} \\
\mathrm{m}^{2}\end{array}$ & $10 \Omega / s q$ & NM & $70-95 \%$ & 1.5 & $\mathrm{~N}$ & $\sim 10 \mathrm{~mm}$ \\
\hline $\begin{array}{c}\text { Dip- } \\
\text { coating }^{42}\end{array}$ & $Y$ & $\begin{array}{l}0.3- \\
2.6 \%\end{array}$ & $50-10^{7} \Omega / s q$ & $1.06-27.6$ & $97-99 \%$ & NM & $\mathrm{N}$ & $\sim 25 \mathrm{~mm}$ \\
\hline H-dip 33 & $\mathrm{~N}$ & $\mathrm{NQ}$ & $2-7 \Omega / s q$ & 1.2 & $60-80 \%$ & $2-3$ & $\mathrm{~N}$ & $\sim 100 \mathrm{~mm}$ \\
\hline $\begin{array}{c}\text { bar- } \\
\text { coating }^{34}\end{array}$ & $Y$ & $\mathrm{NQ}[\mathrm{b}]$ & NA & NA & $80-95 \%$ & $\sim 4$ & $\mathrm{~N}$ & $200 \mathrm{~mm}$ \\
\hline $\begin{array}{l}\text { Microfluidic } \\
\text { s (this work) }\end{array}$ & $Y$ & $\begin{array}{c}10^{4} \mathrm{NW} / \\
\mathrm{mm}^{2}\end{array}$ & $3-5 \Omega / s q$ & $\sim 10$ & $70-80 \%$ & $\sim 25$ & $Y$ & $\begin{array}{l}\sim 5 \mathrm{~mm} \\
\text { wide }\end{array}$ \\
\hline
\end{tabular}

3 Note:

$4 \quad$ Y: Yes; N: No; NM: Not measured; NQ: Not quantified

5 [a] Transmission spectra were measured but the intensities were not quantified

6 [b] Micrographs of nanowire coatings were shown but the areal densities were not quantified.

7 [c] Diameter of the inner surface of the capillary tube

8 

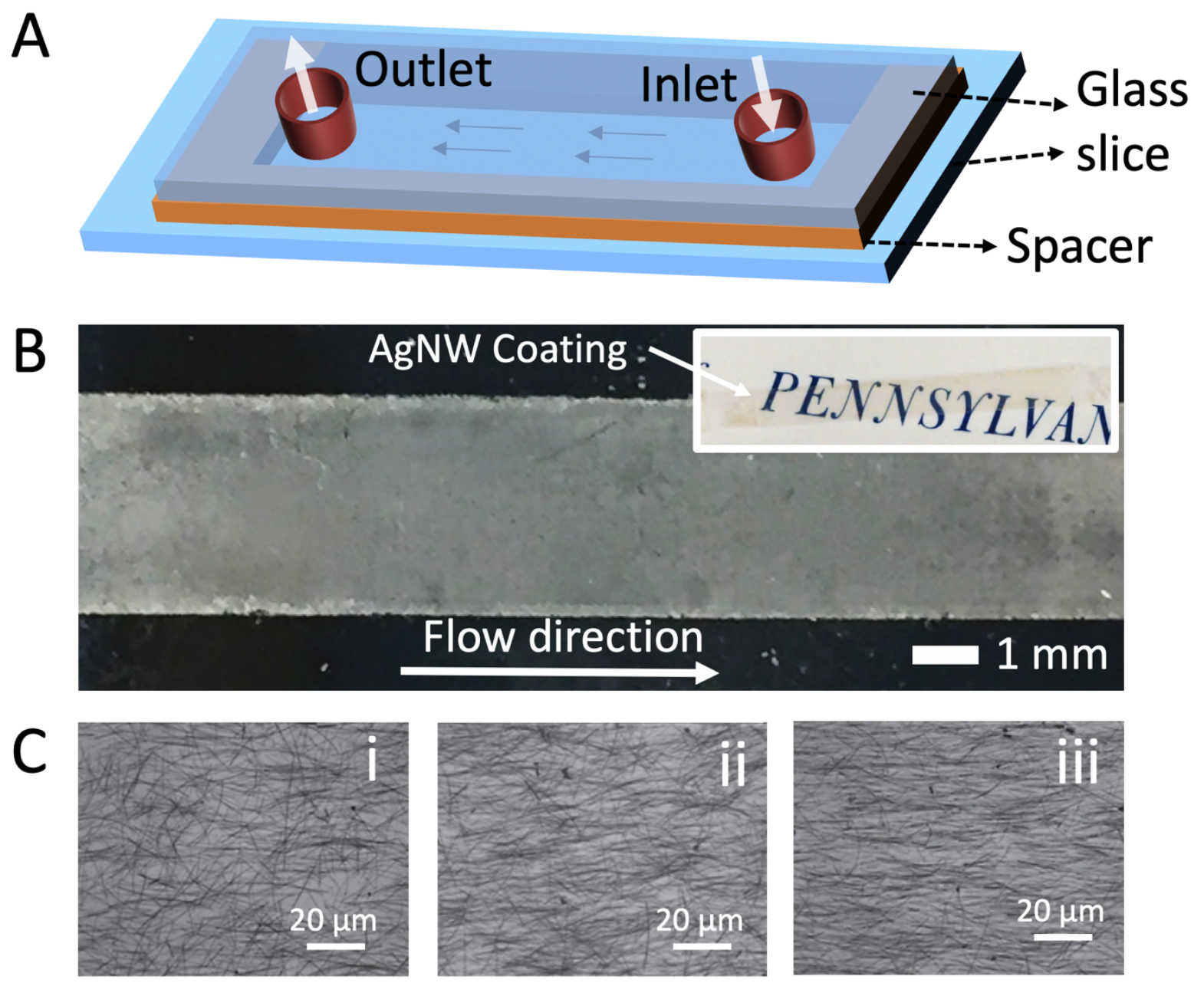

2 Fig. 1: (A) Schematic of flow channels for preparing silver nanowire coatings. (B) Low

3 magnification optical image of the deposited AgNW coating of approximately $40 \%$ areal

4 coverage, showing centimeter-scale uniformity. Inset: Photograph of the same AgNW

5 coating taken on top of the text, showing its transparency. (C) High magnification optical

6 images of AgNW deposited under different flow conditions. The shear rates of AgNW

7 suspension near the glass substrate are $15 \mathrm{~s}^{-1}(\mathrm{i}), 100 \mathrm{~s}^{-1}(\mathrm{ii})$, and $500 \mathrm{~s}^{-1}$ (iii), respectively. 

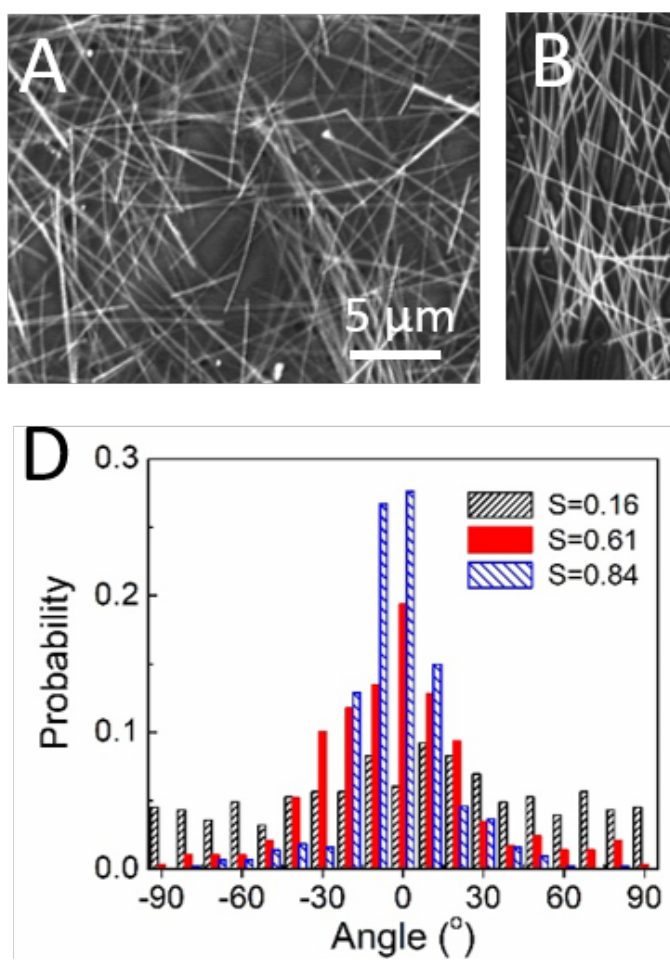
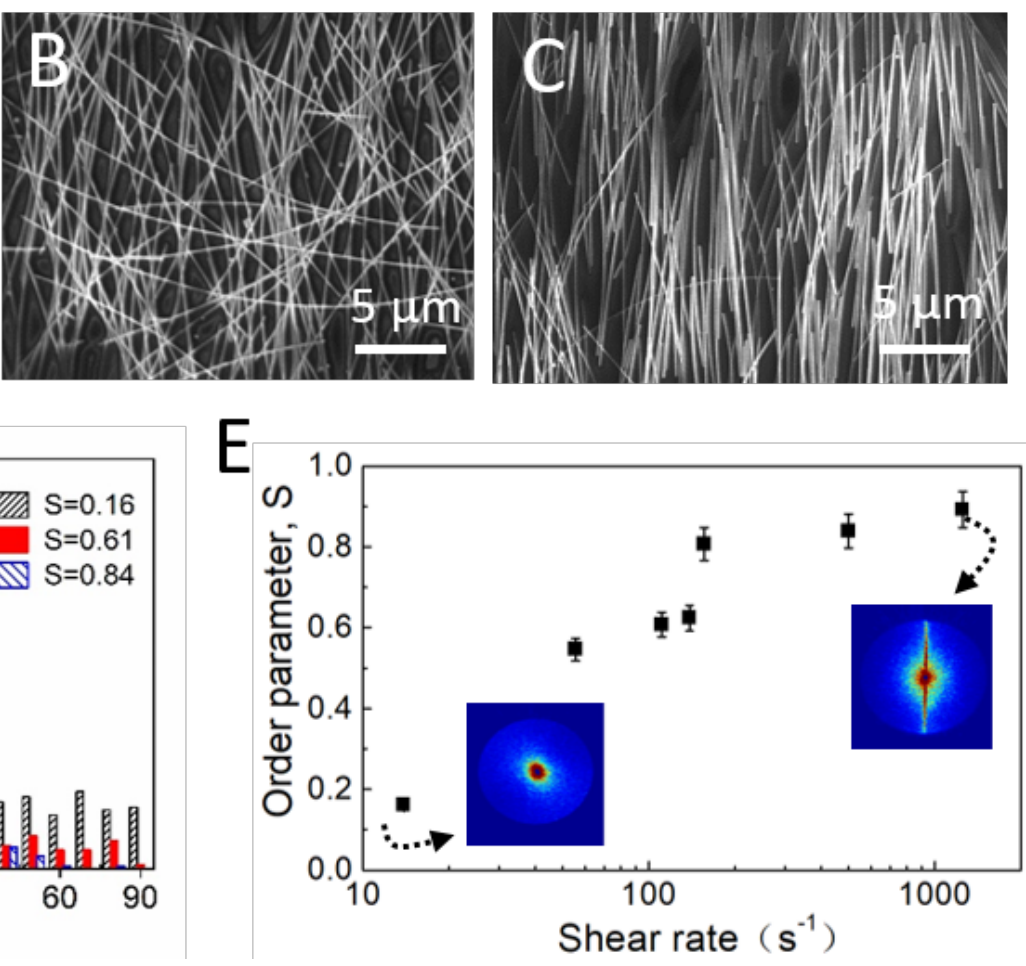

2

Fig. 2: (A-C) SEM images of three AgNW coatings to illustrate analysis of the orientations of

3 individual nanowires. (D) Histogram of the angle between the nanowire orientation and

4 flow direction for three representative samples seen in Fig. 1C with different order

5 parameters: $S=0.16(A),=0.61(B)$, and $0.84(C) .(E)$ Order parameter of deposited AgNW

6 coatings, $S$, as a function of shear rate of the flowing AgNW suspensions near the glass

7 substrate, $\boldsymbol{\gamma}$. Insets: Light scattering patterns of a random and well-aligned AgNW coating

8 in $k$-space. The colors show the intensity of the scattered laser light with red as the highest

$9 \quad$ intensity and blue as the lowest intensity. 

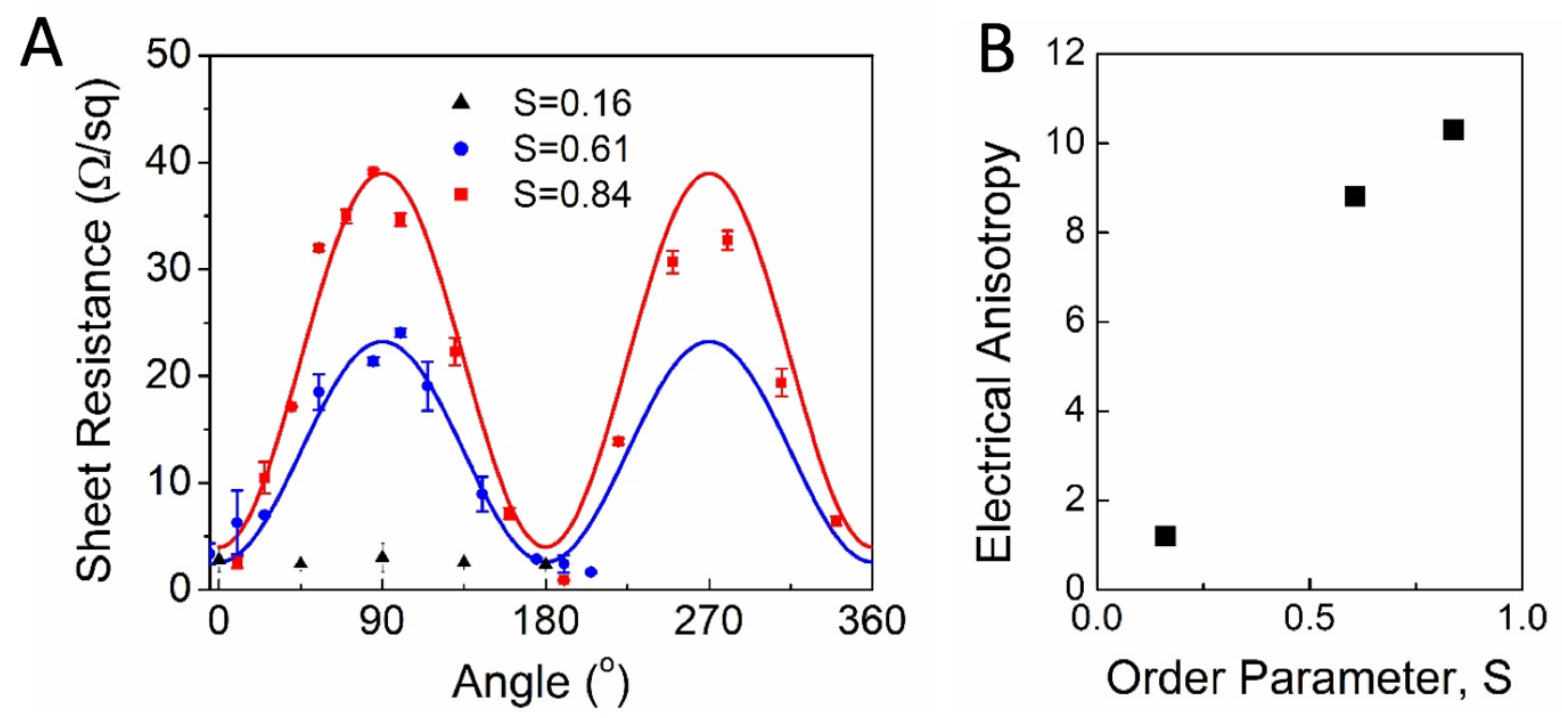

3 Fig. 3: Angle-dependent sheet resistance of the AgNW coatings. (A) Sheet resistance as a

4 function of measurement angle relative to the alignment of the AgNW coatings (i.e., with

5 order parameters of $0.84,0.61,0.16$. Solid lines are fits to a sinusoidal function; (B) Electrical

6 anisotropy as a function of order parameter. 

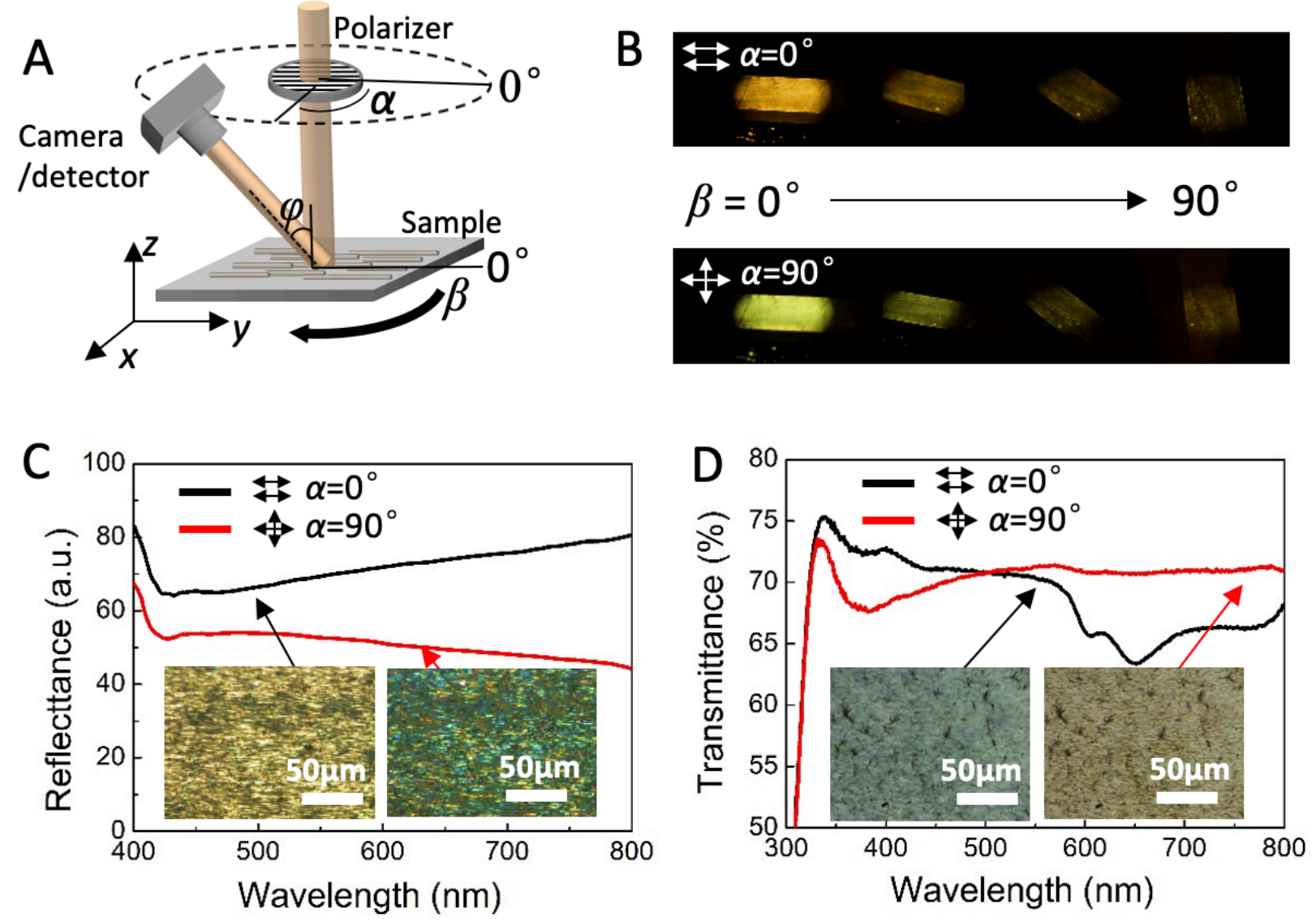

2 Fig. 4: Angle-dependent optical properties of the aligned AgNW coating (S=0.84) under

3 polarized illumination. (A) Schematic of the optical measurement setup showing angles of

4 AgNW alignment $(\boldsymbol{\beta})$, polarization of incident light $(\boldsymbol{\alpha})$, and observation location $(\boldsymbol{\phi})$. (B)

5 Photos taken at $\boldsymbol{\phi}=\mathbf{6 0}^{\circ}$ and various $\beta$ angles when the incident light is polarized parallel

6 (top) and perpendicular (bottom) to the AgNW alignment direction. (C) Spectrum of

7 reflected light for two different input polarization configurations: parallel (black line) and

8 perpendicular (red line) to the AgNW alignment direction. Insets: Optical micrographs of

9 AgNW coating in reflection. (D) Spectrum of transmitted light under parallel and 10 perpendicular illumination. Insets: Optical micrographs of AgNW coating in the transmission 11 mode. 

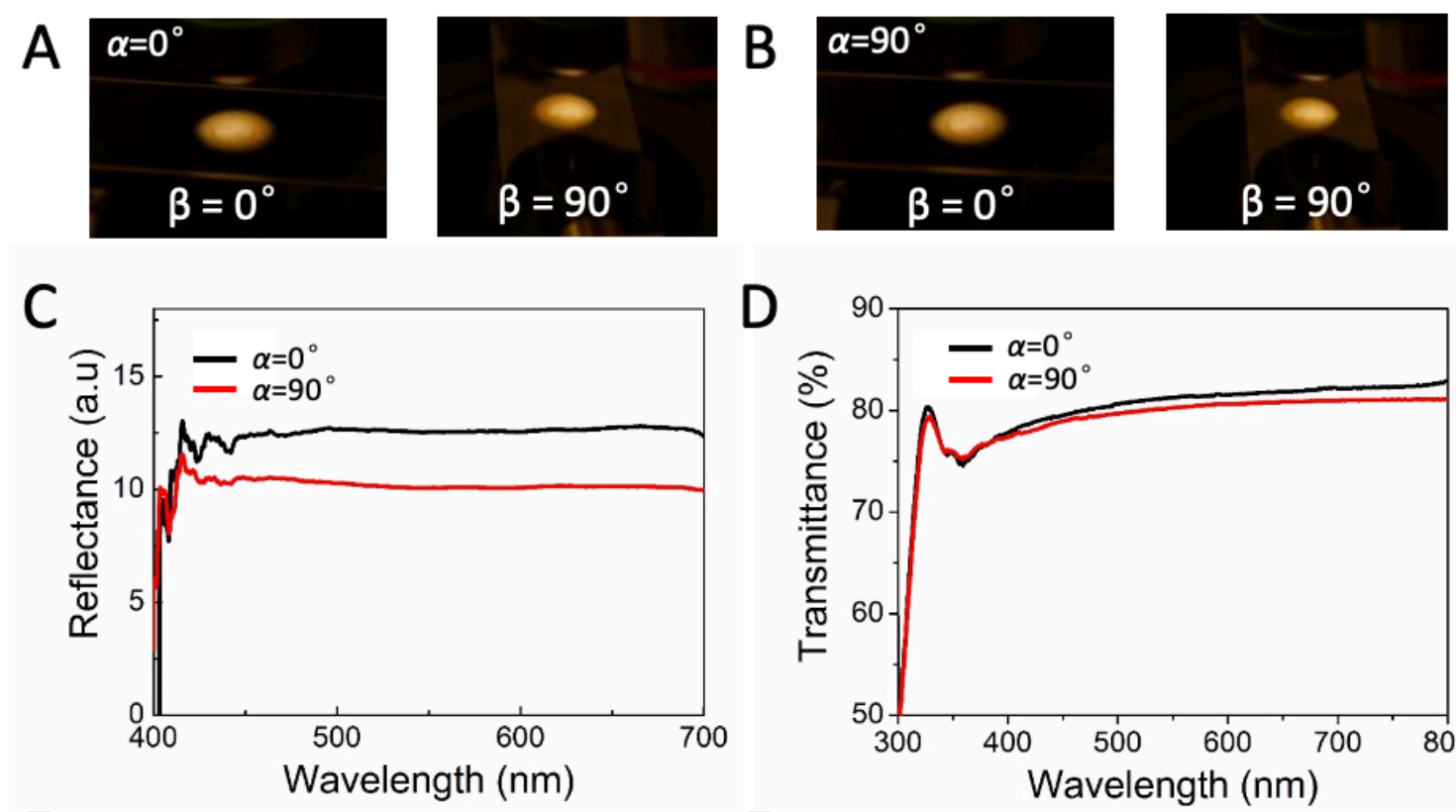

$E$
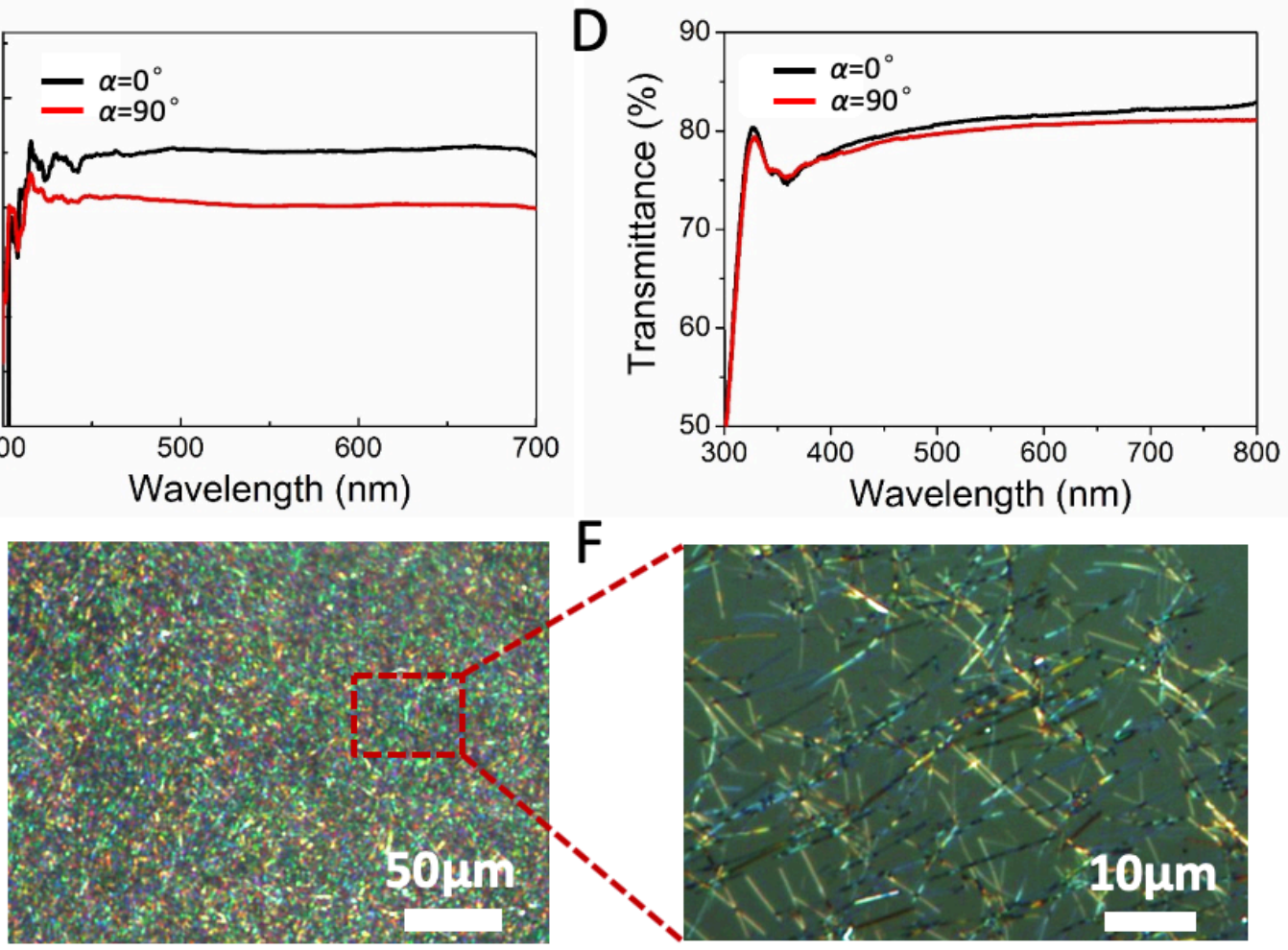

2 Fig. 5: Angle-independent optical appearance and spectrum of randomly oriented AgNW

3 coatings under polarized light. (A) and (B) are photos of a random AgNW coating taken at

4 various $\beta$ angles, under incident polarization of $\boldsymbol{\alpha}=\mathbf{0}^{\circ}$ (A) or $\boldsymbol{\alpha}=\mathbf{0}^{\circ}$ (B) Isotropic coatings

5 appear the same under different polarization and observation angles; (C) and (D) Spectra of

6 reflected and transmitted light by randomly oriented AgNW coatings. (E) Micrograph of a

7 randomly orientated AgNW coating under polarized light showing colors due to distributions

8 of individual nanowires; (F) Zoom-in view showing individual AgNWs that exhibit different

9 colors depending on their orientations. 

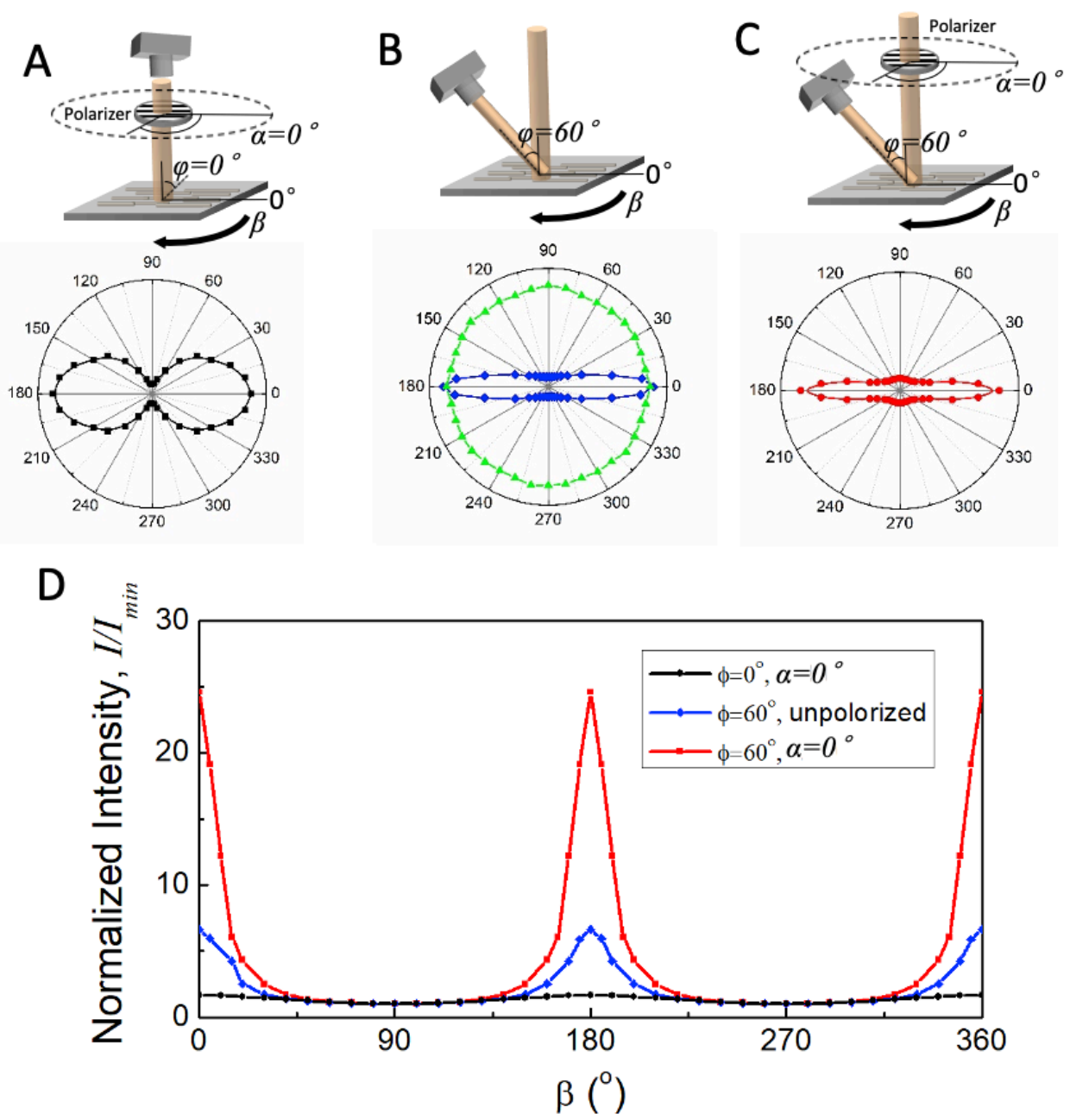

3 Fig. 6: Angle-dependent light intensity from aligned AgNW coatings. (A) Reflected light

4 intensity $\left(\boldsymbol{\phi}=\mathbf{0}^{\circ}\right)$ as a function of $\beta$ angles when the incident light (650nm) is polarized

5 parallel to the AgNW alignment direction $\left(\boldsymbol{\alpha}=\mathbf{0}^{\circ}\right)$; (B) Scattered light intensity $\left(\boldsymbol{\phi}=\mathbf{6 0}^{\circ}\right)$

6 as a function of $\boldsymbol{\beta}$ when a random (green) and well-aligned (blue, $\mathrm{S}=0.84$ ) AgNW coatings are

$7 \quad$ illuminated with unpolarized light; (C) Scattered light intensity $\left(\boldsymbol{\phi}=\mathbf{6 0}^{\circ}\right)$ as a function of $\beta$

8 when the incident light is polarized parallel to the AgNW alignment direction $\left(\boldsymbol{\alpha}=\mathbf{0}^{\circ}\right)$; (D)

9 Normalized light intensity vs. $\boldsymbol{\beta}$ for a well-aligned AgNW ( $\mathrm{S}=0.84)$ coating under different 10 illuminating and observation configurations. 